

EUROPEAN ORGANIZATION FOR NUCLEAR RESEARCH

CERN-PPE/92-130

7 July 1992

\title{
KAON PRODUCTION IN 200 GEV/NUCLEON NUCLEUS-NUCLEUS COLLISIONS
}

\author{
HELIOS Collaboration
}

T. $\AA_{k e s s o n}{ }^{3)}$, S. Almehed ${ }^{17)}$, A.L.S. Angelis ${ }^{20)}$, N.Armenise ${ }^{1)}$, H. Atherton ${ }^{3)}$, P. Aubry ${ }^{8)}$, H.W. Bartels ${ }^{4)}$,G. Beaudoin ${ }^{8)}$, J.M. Beaulieu ${ }^{8)}$, H. Beker ${ }^{3)}$, O. Benary ${ }^{18)}$, D. Bettoni ${ }^{3, a)}$, V. Bisi ${ }^{19)}$, I. Blevis ${ }^{21)}$, H. Bøggild ${ }^{3, b)}$,W. Cleland ${ }^{12)}$, M. Clemen ${ }^{12)}$, B. Collick ${ }^{12)}$, F. Corriveau ${ }^{7)}$, S. Dagan ${ }^{18)}$, K. Dederichs ${ }^{3, c)}$, S. Dell'Uomo ${ }^{13)}$, P. Depommier ${ }^{8)}$, R.C.E. Devenish ${ }^{3, d)}$, S. Di Liberto ${ }^{13)}$, N. DiGiacomo ${ }^{5)}$, J.R. Dodd ${ }^{20)}$, B. Dolgoshein ${ }^{10)}$, A. Drees ${ }^{4}$, H. En'yo ${ }^{3)}$, B. Erlandsson ${ }^{17)}$, M.J. Esten ${ }^{20)}$, C.W. Fabjan $^{3)}$, P. Fischer ${ }^{4)}$, Z. Fraenkel ${ }^{21)}$, A. Gaidot ${ }^{15)}$, F. Gibrat-Debu ${ }^{15)}$, P. Giubellino ${ }^{19)}$, P. Glässel ${ }^{4)}$, U. Goerlach ${ }^{4)}$, R. Haglund ${ }^{6)}$, L.A. Hamel ${ }^{7)}$, H. van Hecke ${ }^{5)}$, V. Hedberg ${ }^{3)}$, R. Heifetz ${ }^{18)}$, A. Hölscher ${ }^{4)}$, B. Jacak ${ }^{5)}$, G. Jarlskog ${ }^{6)}$, S. Johansson ${ }^{6)}$, H. Kraner ${ }^{2)}$, V. $\mathrm{Kroh}^{4)}$, F. Lamarche ${ }^{7)}$, C. Leroy ${ }^{7)}$, D. Lissauer ${ }^{2,18)}$, G. London ${ }^{15)}$, B. Lörstad ${ }^{6)}$, A. Lounis ${ }^{8)}$, F. Martelli19), M. Masera ${ }^{19)}$, A. Marzari-Chiesa ${ }^{19)}$, M.A. Mazzoni ${ }^{3)}$, E. Mazzucato ${ }^{7)}$, M.L. McCubbin ${ }^{20)}$, N.A. McCubbin ${ }^{14)}$, P. McGaughey ${ }^{5)}$, F. Meddi ${ }^{13)}$, U. Mjörnmark ${ }^{6)}$, M.T. Muciaccia ${ }^{1)}$, S. Muraviev ${ }^{9)}$, M. Murray ${ }^{12)}$, M. Neubert ${ }^{4)}$, S. Nilsson ${ }^{17)}$, L. Olsen ${ }^{2}$, Y. Oren ${ }^{18)}$, J.P. Pansart ${ }^{15)}$, Y.M. Park ${ }^{12)}$, A. Pfeiffer ${ }^{4)}$, F. Piuz ${ }^{3)}$, V. Polychronakos ${ }^{2)}$, G. Poulard ${ }^{3)}$, M. Price ${ }^{3)}$, D. $\operatorname{Rahm}^{2)}$, L. Ramello ${ }^{19}$, L. Riccati ${ }^{19)}$, G. Romano ${ }^{16)}$, G. Rosa ${ }^{13)}$, L. $\operatorname{Sandor}^{3)}$, J. Schukraft ${ }^{3)}$, M. Sekimoto ${ }^{3, e)}$, B. Sellden ${ }^{17)}$, M. Seman ${ }^{3, f)}$, A. Shmeleva ${ }^{9)}$, V. Sidorov ${ }^{11)}$, S. Simone ${ }^{1)}$, Y. Sirois ${ }^{7)}$, H. Sletten ${ }^{3)}$, S. Smirnov ${ }^{10)}$, J. Soltani ${ }^{4)}$, W. Sondheim ${ }^{5)}$, H. J. Specht4), E. Stern ${ }^{12)}$, I. Stumer ${ }^{2)}$, J. Sunier ${ }^{5)}$, V. Tcherniatin ${ }^{10)}$, H. H. Thodberg ${ }^{3)}$, J. Thompson ${ }^{12)}$, V. Tikhomirov ${ }^{9)}$, I. Tserruya ${ }^{21)}$, G. Vasseur ${ }^{15)}$, R. Veenhof ${ }^{3, g)}$, R. Wigmans ${ }^{3, g)}$ and P. Yepes ${ }^{7)}$

\section{Abstract}

Results from the HELIOS External Spectrometer on kaon production in 200 $\mathrm{GeV} / \mathrm{A} \mathrm{S}+\mathrm{W}$ and $\mathrm{p}+\mathrm{W}$ collisions are presented. The $K / \pi$ ratios are compared with results from a lower beam energy and are found to be remarkably similar. Evidence for secondary production of $\mathrm{K}^{+}$by meson-baryon rescattering is reviewed. Our results at $y=1.0-1.5$ are compared with neutral strange particle results at midrapidity. 
1) University of Bari and INFN, Bari, Italy

2) Brookhaven National Laboratory, Upton, NY, USA

3) CERN, Geneva, Switzerland

4) Heidelberg University, Fed. Rep. Germany

5) Los Alamos National Laboratory, Los Alamos, NM, USA

6) Lund University, Sweden

7) McGill University, Montreal, Canada

8) University of Montreal, Canada

9) Lebedev Physics Institute, Moscow, USSR

10) Physics Engineering Institute, Moscow, USSR

11) Institute Nuclear Physics, Novosibirsk, USSR

12) University of Pittsburgh, Pittsburgh, PA, USA

13) University of Rome and INFN, Rome, Italy

14) Rutherford Appleton Laboratory, Didcot, UK

15) DPhPE, CEN-Saclay, Gif-sur-Yvette, France

16) University of Salerno and INFN, Salerno, Italy

17) Stockholm University, Sweden

18) Tel Aviv University, Israel

19) University of Turin and INFN, Turin, Italy

20) University College, London, UK

21) Weizmann Institute, Rehovot, Israel

Visitors at CERN from:

a) Syracuse University, Syracuse, NY, USA

b) Niels Bohr Institute, Copenhagen, Denmark

c) University of Munich, Fed. Rep. Germany

d) Oxford University, UK

e) Institute of Nuclear Study, Tokyo, Japan

f) Slovak Academy of Sciences, Kosice, Czechoslovakia

g) NIKHEF-H, Amsterdam, The Netherlands 


\section{Introduction}

A possible signature of deconfinement of quarks in ultra-relativistic heavy ion collisions is enhanced production of strange particles. ${ }^{1}$ In an equilibrated quark-gluon plasma, the density of strange quarks and antiquarks may be comparable to that of the non-strange quarks. Upon cooling and hadronization of the plasma, these would result in an enhanced yield of strange particles. However, the process of hadronization can affect the final number of strange particles ${ }^{2,3}$ and complicate the picture. It is also possible that non-equilibrium effects may cause strange particle production in relativistic heavy ion reactions to exceed expectations from p-p collisions regardless of whether deconfinement takes place. ${ }^{4}$

Comparison of $\mathrm{K}^{+}$and $\mathrm{K}^{-}$momentum spectra may provide a probe of the collision dynamics, particularly if an initial plasma was formed in a baryon-rich environment. ${ }^{2}$ Because of the greater abundance of $u$ and $d$ quarks (as compared to $\bar{u}$ and $\bar{d}$ quarks), the mean free path of the $\bar{s}$ quark in the hadronizing system is shorter than that of the $s$ quark. This causes $K^{+}$and hyperons to form earlier in the hadronization process than $K^{-}$and anti-hyperons. Then, any change in the condition of the plasma during hadronization, such as a change of temperature, would cause a difference in the slope ("temperatiure") of the $K^{+}$and $K^{-}$spectra. It is of interest to study both the rapidity density and energy spectra of $K^{+}$and $K^{-}$in a baryon-rich environment of heavy ion collisions. At $200 \mathrm{GeV} / \mathrm{A}$ the target rapidity region, $y=1.0-1.5$, presents an environment with many baryons. The $K^{+}$and $K^{-}$spectra may be compared with the E802 collaboration results ${ }^{5}$ at $14.6 \mathrm{GeV} / \mathrm{A}$, where the dynamics of the reaction and the amount of stopping should be quite different. Our data complement the measurement by NA35 of $K_{s}^{0}$ and $\Lambda$ produced at midrapidity in $\mathrm{p}+\mathrm{Au}, \mathrm{O}+\mathrm{Au}, \mathrm{p}+\mathrm{S}$ and $\mathrm{S}+\mathrm{S}$ reactions at $200 \mathrm{GeV} / \mathrm{A}^{6,7}$

\section{Experiment}

We have measured $K, \pi$, and $p$ distributions at $y=1.0-1.5$ in $\mathrm{p}+\mathrm{W}$ and $\mathrm{S}+\mathrm{W}$ collisions at 200 $\mathrm{GeV} / \mathrm{A}$ using the HELIOS external spectrometer ${ }^{8,9}$. The spectrometer consists of a magnet flanked by two drift chambers, and an array of time-of-flight scintillators. The spectrometer views the target through a $10 \mathrm{~cm}$ high slit in one of the HELIOS calorimeter walls, extending from 15 to 45 degrees with respect to the beam direction. Event characterization is provided by the transverse energy measured in the HELIOS calorimeters, which cover the full solid angle of $4 \pi$ with good granularity.

Particles are identified using two time-of-flight scintillator hodoscopes 5 meters from the target; the time resolution is 250 ps. Tracks in the drift chambers are matched to the scintillator hits, and the square of the mass is calculated from the reconstructed momentum and the flight time. The mass-squared distribution was analyzed for $100 \mathrm{MeV} / \mathrm{c}$ intervals in transverse momentum, $p_{t}$, and the number of pions and kaons was extracted by simultaneously fitting gaussian distributions to the pion, kaon and proton peaks in these spectra. A mass-squared distribution along with the fit is shown as figure 2 in reference 9 . The relative numbers of pions and kaons from the fits yield the $K / \pi$ ratios. 
A Monte Carlo simulation of the detector was done to correct for geometrical acceptance, detection and reconstruction efficiency, multiple scattering and particle decay. The Monte Carlo generator produced pions and kaons with relative numbers and $p_{t}$ distributions taken from $p-p$ data ${ }^{10}$. The events were analyzed in the same fashion as the data, and the results presented below include these corrections. Details of the calibration procedures and the Monte Carlo simulation are given in reference 9 .

The systematic errors are dominated by uncertainties in the fitting procedure. These were studied by examining the dependence of the results on assumptions for the peak shapes. Also, results were compared for several independent fits with the values of various parameters fixed, for example the relative widths and peak positions of the gaussians. Other systematic errors cancel when $K / \pi$ ratios are constructed.

\section{Results}

Figure 1 shows the $\mathrm{K}^{+} / \pi^{+}$and $\mathrm{K}^{-} / \pi^{-}$ratios as a function of $p_{t}$ in $\mathrm{S}+\mathrm{W}$ collisions. The inner error bars indicate the statistical errors and the outer error bars statistical and systematic errors added in quadrature. The geometrical acceptance of the spectrometer and the time-of-flight resolution limit the analysis to a rapidity interval $y=1.0-1.5$ and transverse momentum region $p_{t}=200-700 \mathrm{MeV} / \mathrm{c}$. The dotted lines represent $\mathrm{K}^{+} / \pi^{+}$and the dashed lines $K^{-} / \pi^{-}$ratios from p-p data ${ }^{10}$. These data are scaled to the proper $y$ interval using the rapidity distributions for pions and kaons published ${ }^{10}$ at $p_{t}=0.4 \mathrm{GeV} / \mathrm{c}$, which is approximately the mean $p_{t}$ for $\pi$ and $K$. The scaling to our rapidity region introduces an uncertainty, which is indicated by the error bar on the right of the dashed line. In both $\mathrm{p}+\mathrm{p}$ and $\mathrm{S}+\mathrm{W}$ collisions, the $K / \pi$ ratios increase with $p_{t}$. A clear excess in $K^{+} / \pi^{+}$is seen in $\mathrm{S}+\mathrm{W}$, compared to $\mathrm{p}+\mathrm{p}$. No such excess is present for $\mathrm{K}^{-} / \pi^{-}$in the $p_{t}$ interval under study.

To investigate the dependence of this excess on impact parameter, we subdivided our data into a 'low $E_{t}$ ' sample with $E_{t}(-0.1<\eta<2.9)<80 \mathrm{GeV}$, representing peripheral collisions, and a 'high $E_{t}$ ' sample with $E_{t}(-0.1<\eta<2.9)>180 \mathrm{GeV}$, containing the most central $\mathrm{S}+\mathrm{W}$ collisions. Combining the data in the $p_{t}$ interval from 200 to $700 \mathrm{MeV} / \mathrm{c}$ yields the values shown in Table 1 . We see no indication for a significant relative increase in kaon production in high $E_{t}$ over low $E_{t}$ events in our $p_{t}$ - $y$ domain. Though one might expect peripheral nucleus-nucleus collisions to resemble p-p collisions, it is important to note that the low $E_{t}$ selection does not correspond to only a single nucleon-nucleon collision, but rather represents a range of impact parameters. The presence of the spectator nucleons is important in these events. It is remarkable that the ratios measured in $200 \mathrm{GeV} / \mathrm{A} \mathrm{S}+\mathrm{W}$ are nearly identical to those measured by the E802 collaboration ${ }^{5}$ in $14.6 \mathrm{GeV} / \mathrm{A} \mathrm{Si}+\mathrm{Au}$.

We can investigate effects arising from spectator matter, such as rescattering ${ }^{4}$, by comparing the $\mathrm{S}+\mathrm{W}$ and $\mathrm{p}+\mathrm{W}$ results. In Fig. 2, the $K / \pi$ ratios for $200 \mathrm{GeV} \mathrm{p}+\mathrm{W}$ are plotted as a function of $p_{t} . K^{+} / \pi^{+}$in these data is also enhanced over p-p collisions, but the excess is smaller. Combining the data in figures 1 and 2 over the entire $p_{t}$ interval yields $K / \pi(\mathrm{S}+\mathrm{W}) / K / \pi(\mathrm{p}+\mathrm{W})=1.24 \pm 0.09$ for 


\begin{tabular}{cccccc}
\hline & $\begin{array}{c}\mathrm{S}+\mathrm{W} \\
\text { low } E_{t}\end{array}$ & $\begin{array}{c}\mathrm{S}+\mathrm{W} \\
\text { high } E_{t}\end{array}$ & $\begin{array}{c}\mathrm{Si}+\mathrm{Au}^{5} \\
\text { central }\end{array}$ & $\mathrm{p}+\mathrm{W}$ & \multirow{2}{*}{$\mathrm{pp}^{11 *}$} \\
\hline \hline$K^{+} / \pi^{+}$ & $17.6 \pm 1.5 \%$ & $19.7 \pm 0.9 \%$ & $19.2 \pm 3 \%$ & $14.1 \pm 0.8$ & $10.8 \pm 0.9 \%$ \\
$K^{-} / \pi^{-}$ & $5.0 \pm 0.6 \%$ & $5.3 \pm 0.4 \%$ & $3.6 \pm 0.8 \%$ & $3.7 \pm 0.4$ & $8.6 \pm 0.8 \%$ \\
\hline
\end{tabular}

$* \sqrt{s}=23 \mathrm{GeV}$, midrapidity, $p_{t}=200-700 \mathrm{MeV} / \mathrm{c}$

positives and $1.31 \pm 0.22$ for negatives. The presence of the increase in both $p+W$ and $S+W$ suggests it does not indicate significant new effects in nucleus-nucleus compared to proton-nucleus collisions. An increase in $\mathrm{K}^{+}$production from $\mathrm{p}+\mathrm{p}$ to $\mathrm{p}+\mathrm{W}$ collisions is expected from rescattering in the target matter ${ }^{7}$ and other non-equilibrium effects. ${ }^{4}$

Figure 3 shows the transverse mass $\left(m_{t}=\sqrt{p_{t}^{2}+m_{0}^{2}}\right)$ spectra of kaons, negative pions and protons in $S+W$ collisions. The distributions of the different particle species are normalized relative to one another. Only $\pi^{-}$are shown in Figure 3 since $\pi^{+}$and $\pi^{-}$have similar distributions. Note that the pion low $p_{t}$ excess $^{8}$ is clearly visible. The proton distribution is taken from reference 9 .

The distributions have been fit with exponentials in the $m_{t}$ ranges corresponding to $200 \mathrm{MeV} / \mathrm{c}$ $<p_{t}<600 \mathrm{MeV} / \mathrm{c}$ for kaons, $300 \mathrm{MeV} / \mathrm{c}<p_{t}<700 \mathrm{MeV} / \mathrm{c}$ for pions (omitting the region of the low $p_{t}$ excess), and $450 \mathrm{MeV} / \mathrm{c}<p_{t}<825 \mathrm{MeV} / \mathrm{c}$ for protons. The resulting inverse slopes are given in Table 2, along with values for $p-p$, and nucleus-nucleus measurements from other experiments. The $\mathrm{p}-\mathrm{W}$ spectra have not been fit due to the large statistical errors in that data set. Some caution must be used in comparing the values, as the slopes are fitted over different ranges in $m_{t}$ in the different experiments. However, the general trends may be determined.

The $K^{+}$distribution appears slightly flatter than $K^{-}$in both heavy ion data sets. The $K_{s}^{0}$ slope of $193 \mathrm{MeV}$, reported by NA35 for central S+S collisions, ${ }^{7}$ falls in between the $K^{+}$and $K^{-}$ slopes. In p-p collisions, ${ }^{11}$ the difference between $K^{+}$and $K^{-}$is smaller. There, the $K^{+}$distribution is flattest, the proton distribution is steepest, and the $K^{-}$and $\pi$ fall in between. In both heavy ion data sets, the proton distributions are not nearly as steep. An increase in the proton $m_{t}$ slope over that in $\mathrm{p}-\mathrm{p}$ collisions is expected if significant rescattering takes place. ${ }^{12}$

\section{Discussion}

The rapidity density of protons measured by HELIOS near $y=1$, where the $K / \pi$ ratios are reported, rises approximately linearly with $E_{t}$ for all projectiles. ${ }^{9}$ In central $\mathrm{S}+\mathrm{W}$ collisions, more than 15 protons per unit rapidity are observed. A very similar proton rapidity density was reported by $\mathrm{E} 802^{5}$ at $y \sim 1.3$, where they also measure $K / \pi$. It should be noted that in both experiments, the 
TABLE 2 - INVERSE SLOPES OF EXPONENTIAL FITS TO $M_{T}$ DISTRIBUTIONS

\begin{tabular}{|c|c|c|c|}
\hline & $\mathrm{S}+\mathrm{W} 200 \mathrm{GeV} / \mathrm{A}$ & $\mathrm{Si}+\mathrm{Au}^{5} 14.6 \mathrm{GeV} / \mathrm{A}$ & $\mathrm{pp}^{11} \sqrt{s}=23$ \\
\hline$y$ range & 1.1 .5 & 1.3 & 3 \\
\hline $\mathrm{K}+$ & $268 \pm 91 \mathrm{MeV}$ & $203 \mathrm{MeV}$ & $150 \mathrm{MeV}$ \\
\hline $\mathrm{K}-$ & $136 \pm 56$ & 175 & 141 \\
\hline pi- & $209 \pm 42$ & 161 & 141 \\
\hline \multirow[t]{2}{*}{$\mathrm{p}$} & $175 \pm 7$ & 215 & 108 \\
\hline & $\mathrm{S}+\mathrm{S}^{7} 200 \mathrm{GeV} / \mathrm{A}$ & $\mathrm{O}+\mathrm{Au}^{6} 200 \mathrm{GeV} / \mathrm{A}$ & \\
\hline$y$ range & $1-2.5$ & $1.5-2.5$ & \\
\hline$K_{s}^{0}$ & $193 \mathrm{MeV}$ & $238 \mathrm{MeV}$ & \\
\hline$\Lambda$ & 194 & 204 & \\
\hline $\bar{\Lambda}$ & 194 & $313 \pm 71$ & \\
\hline
\end{tabular}

rapidity density of positive pions (and also of negative pions) is equal to the proton rapidity density in the region studied. Though there is a significant difference in the $\sqrt{s}$ of the two experiments, they measure $K / \pi$ in environments with similar numbers of surrounding hadrons. The similarity of the observed $K / \pi$ ratios suggests that the number of kaons depends on the surrounding particles. This is exactly to be expected if the $K^{+}$excess arises from secondary production in rescattering. It is important to note that NA35 sees a large $\Lambda$ yield at $y<y_{\mathrm{cm}}$ in proton-nucleus ${ }^{7}$ and $\mathrm{O}+\mathrm{Au}$ collisions. ${ }^{6}$ Cascading processes such as $\pi+N \rightarrow \Lambda+K^{+}$and $K^{-}+N \rightarrow \Lambda+\pi$ will enrich both the $\Lambda$ and $K^{+}$populations in a baryon-rich environment. This result along with the $K^{+}$enhancement reported here represents a challenge to models incorporating rescattering effects. They must correctly account for the baryon densities as well as the $K$ and $\Lambda$ yields in collisions at both SPS and AGS energies.

The ratio $\left(K^{+}+K^{-}\right) /\left(\pi^{+}+\pi^{-}\right)$is $12 \%$ for $\mathrm{S}+\mathrm{W}$ and $8.9 \%$ for $\mathrm{p}+\mathrm{W}$ collisions from HELIOS. These may be compared to the $K_{s}^{0} / \pi^{-}$ratio reported by $\mathrm{NA}^{7} 5^{7}$ of $11 \%$ for $\mathrm{S}+\mathrm{S}$ and $6 \%$ for $\mathrm{p}+\mathrm{S}$. Though the charged kaon measurement was made near the target rapidity where cascading could be important and the neutral kaon measurement was at midrapidity, the results are quite similar. The $p_{t}$ ranges of the measurements are quite different, however; the midrapidity ratio is extrapolated from a measurement above $625 \mathrm{MeV} / \mathrm{c}$, while the target region measurement does not extend above $p_{t}=$ $700 \mathrm{MeV} / \mathrm{c}$. The $K / \pi$ ratio depends strongly on $p_{t}$ in p-p collisions. ${ }^{10}$

If collective flow takes place in $\mathrm{S}+\mathrm{W}$ collisions, a mass dependent boost of the transverse momenta of the hadrons is expected. This boost complicates the comparison of $K^{+}$and $K^{-}$distributions because particles decoupling early are not accelerated by collective outward flow, whereas late decoupling particles are. In a thermal system with no phase transition to quark matter, the $\mathrm{K}^{+}$would sample an earlier time in the expansion and cooling of the hadronic matter, as mentioned above, and 
should therefore exhibit a flatter $p_{t}$ or $m_{t}$ distribution than the $\mathrm{K}^{-}$, which decouple later. Within errors, our measured $\pi, K$ and $p$ slopes do not show the increase with mass expected from a strong collective flow. For systems without collective flow, Heinz, et al. ${ }^{2}$ predict that if quark matter is formed during the early part of the collision, the $\mathrm{K}^{+}$and $\mathrm{K}^{-}$slopes should be very similar; the $\mathrm{K}^{+}$ distribution may even be somewhat steeper than $\mathrm{K}^{-}$. Our data show a difference between the $\mathrm{K}^{+}$ and $K^{-}$slopes, though the large statistical errors make it difficult to draw a firm conclusion.

\section{Conclusion}

We have measured $K / \pi$ ratios in the target rapidity region, $y=1.0-1.5$, in $200 \mathrm{GeV} / \mathrm{A} \mathrm{S}+\mathrm{W}$ and $\mathrm{p}+\mathrm{W}$ collisions. A significant enhancement of $K^{+}$production is observed in $\mathrm{S}+\mathrm{W}$, and a small enhancement in $\mathrm{p}+\mathrm{W}$. The relative abundances of the different species imply that the number of baryons appears to have an important effect on $K^{+} / \pi^{+}$. Secondary $K^{+}$production from rescattering could cause the observed excess; rescattering could also produce a flatter proton $m_{t}$ distribution than that observed in p-p collisions. Interpretation of these results will require predictions of the hadron distributions in $p_{t}$ and $y$, as well as their relative abundances, from models describing the dynamics of these collisions.

\section{Acknowledgements}

The HELIOS Collaboration acknowledges the outstanding performance of the PS-SPS accelerator complex. We are grateful for support by the Natural Science and Engineering Research Council of Canada, the Institut de Recherche Fondamentale (CEA, France), the German Federal Minister for Research and Technology, the U.S.-Israel Binational Science Foundation, the Istituto Nazionale di Fisica. Nucleare of Italy, the Scientific Reserach Council of Sweden, the Science Research Council of the United Kingdom, and the US Department of Energy.

\section{References}

1. P.Koch, B.Mueller, and J.Rafelski, Phys.Rep. 142 (1987) 176.

2. U.Heinz, K.S.Lee and M.J.Rhoades-Brown, Phys.Rev.Lett. 58 (1987) 2292; K.S.Lee, M.J.RhoadesBrown and U.Heinz, Phys.Rev. C37 (1988) 1463.

3. H.W.Barz, B.L.Friman, J.Knoll and H.Schulz, Nucl.Phys. A484 (1988) 661.

4. R.Mattielo, H.Sorge, H.Stoecker and W.Greiner, Phys.Rev.Lett. 63 (1989) 1459.

5. T.Abbott et al., E802 collaboration, Phys.Rev.Lett. 64 (1990) 847.

6. A.Bamberger et al., NA35 collaboration, Z.Phys. C43 (1989) 25.

7. A.Bartke et al., NA35 collaboration, Z.Phys. C48 (1990) 191.

8. T. Åkesson, et al., HELIOS collaboration, Z. Phys. C46 (1990) 361.

9. T. Åkesson, et al., HELIOS collaboration, Z. Phys. C53 (1992) 183.

10. B. Alper, et al., Nucl. Phys. B100 (1975) 237. 
11. K. Guettler, et al., Phys. Lett. 64B (1976) 111; K. Guettler, et al, Nucl. Phys. B116 (1976) 77.

12. M. Neubert, G. Baym, G. Friedman, B.V. Jacak, and Y. Yariv, to be published. 


\section{Figure Captions}

Fig. 1. $K / \pi$ ratios measured at $y=1.0-1.5$, as a function of $p_{t}$ for $200 \mathrm{GeV} / \mathrm{A} \mathrm{S}+\mathrm{W}$ collisions. The data sample contains peripheral and central collisions, but is biased somewhat toward central collisions by the trigger requirements. The dotted lines represent $\mathrm{K}^{+} / \pi^{+}$and the dashed lines $\mathrm{K}^{-} / \pi^{-}$ratios from $p-p$ data ${ }^{10}$. The inner error bars show statistical errors, and the outer ones include systematic errors also.

Fig. 2. $K / \pi$ ratios at $y=1.0-1.5$, as a function of $p_{t}$ for $200 \mathrm{GeV} \mathrm{p}+\mathrm{W}$ collisions.

Fig. 3 Distribution of the invariant cross section at $y=1.0-1.5$ as a function of transverse mass $m_{t}=\sqrt{p_{t}^{2}+m_{0}^{2}}$, for $\pi, \mathrm{K}$ and $\mathrm{p}$ from $200 \mathrm{GeV} / \mathrm{A} \mathrm{S}+\mathrm{W}$ collisions. The error bars show statistical errors only. 


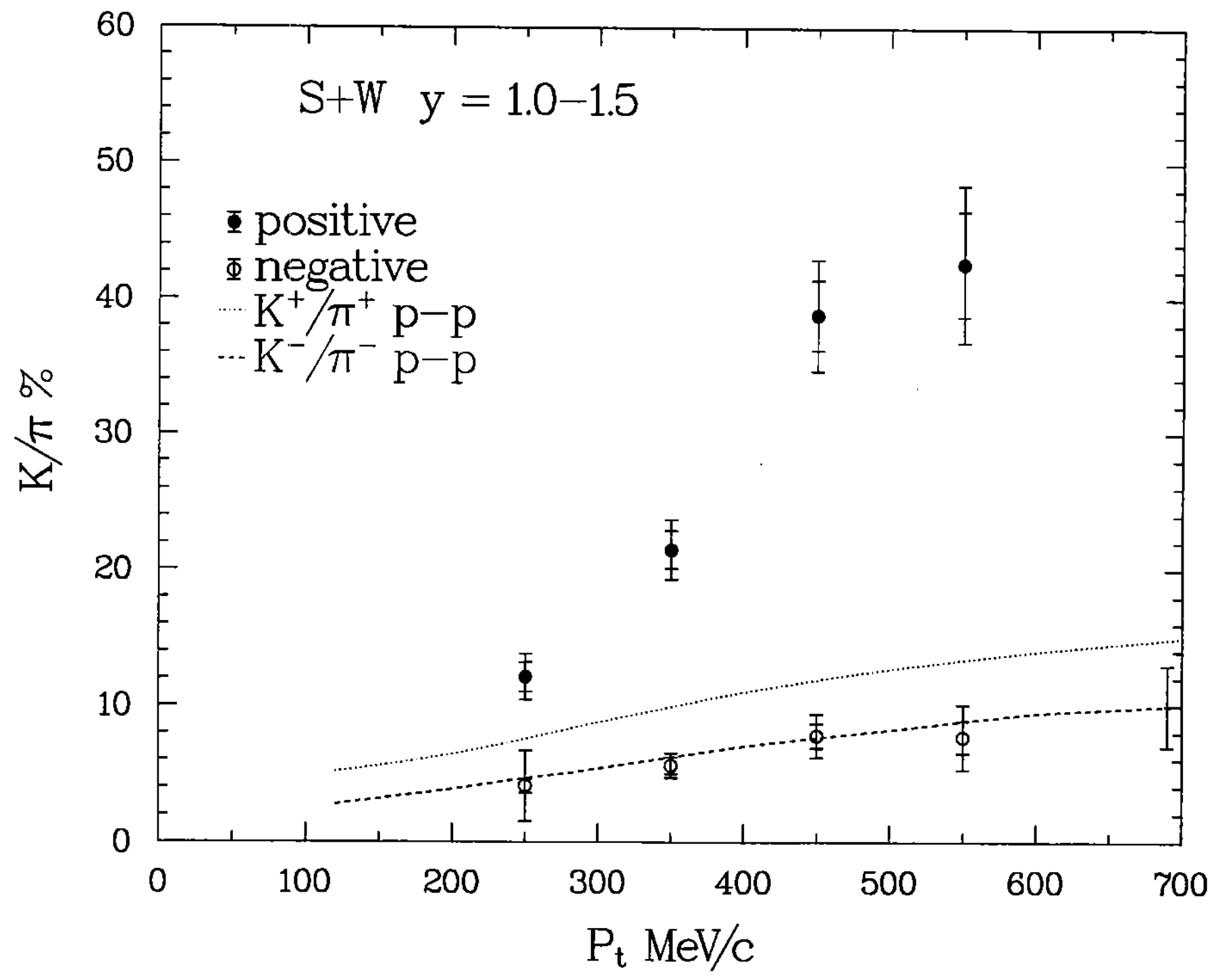

Fig. 1 


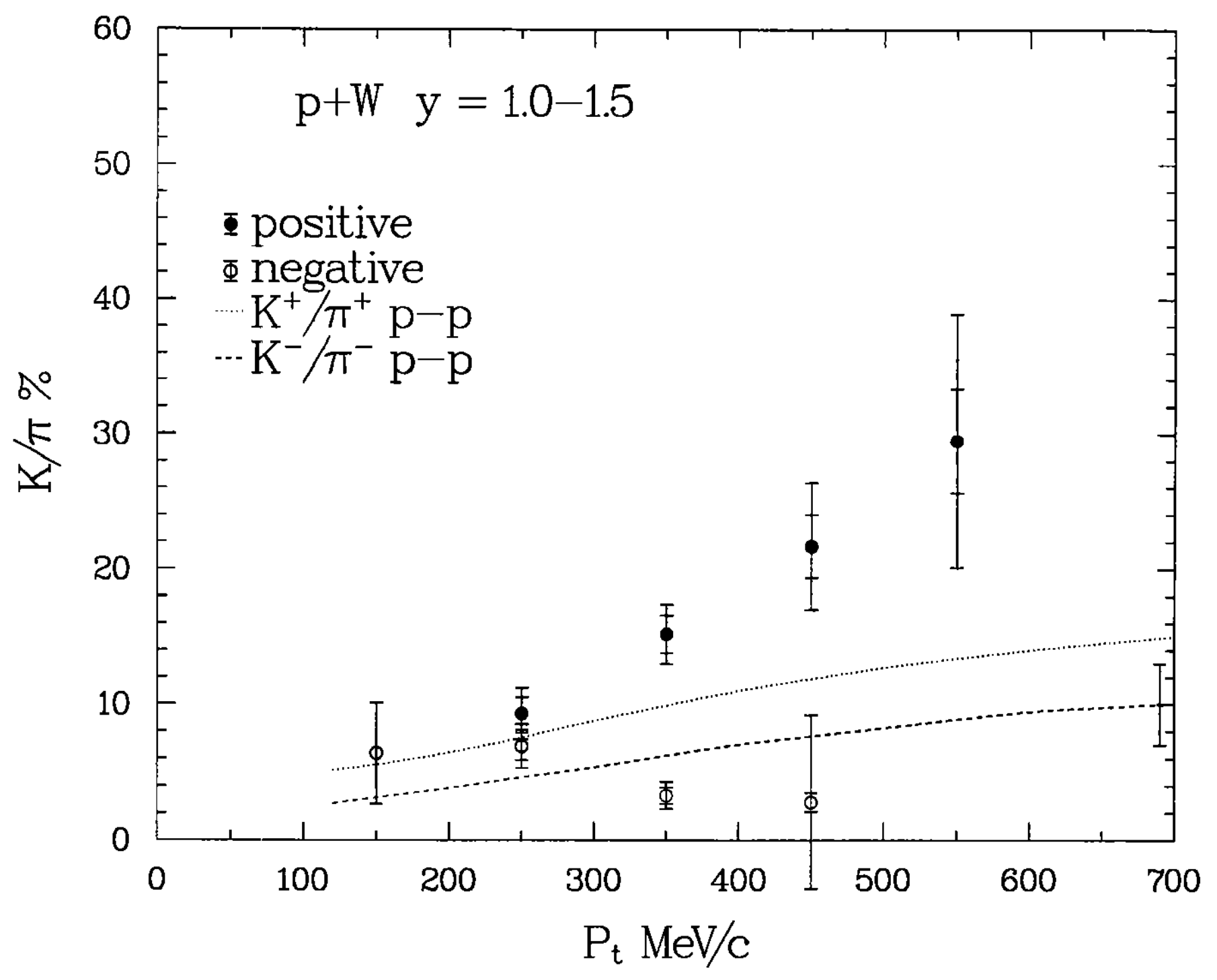

Fig. 2 


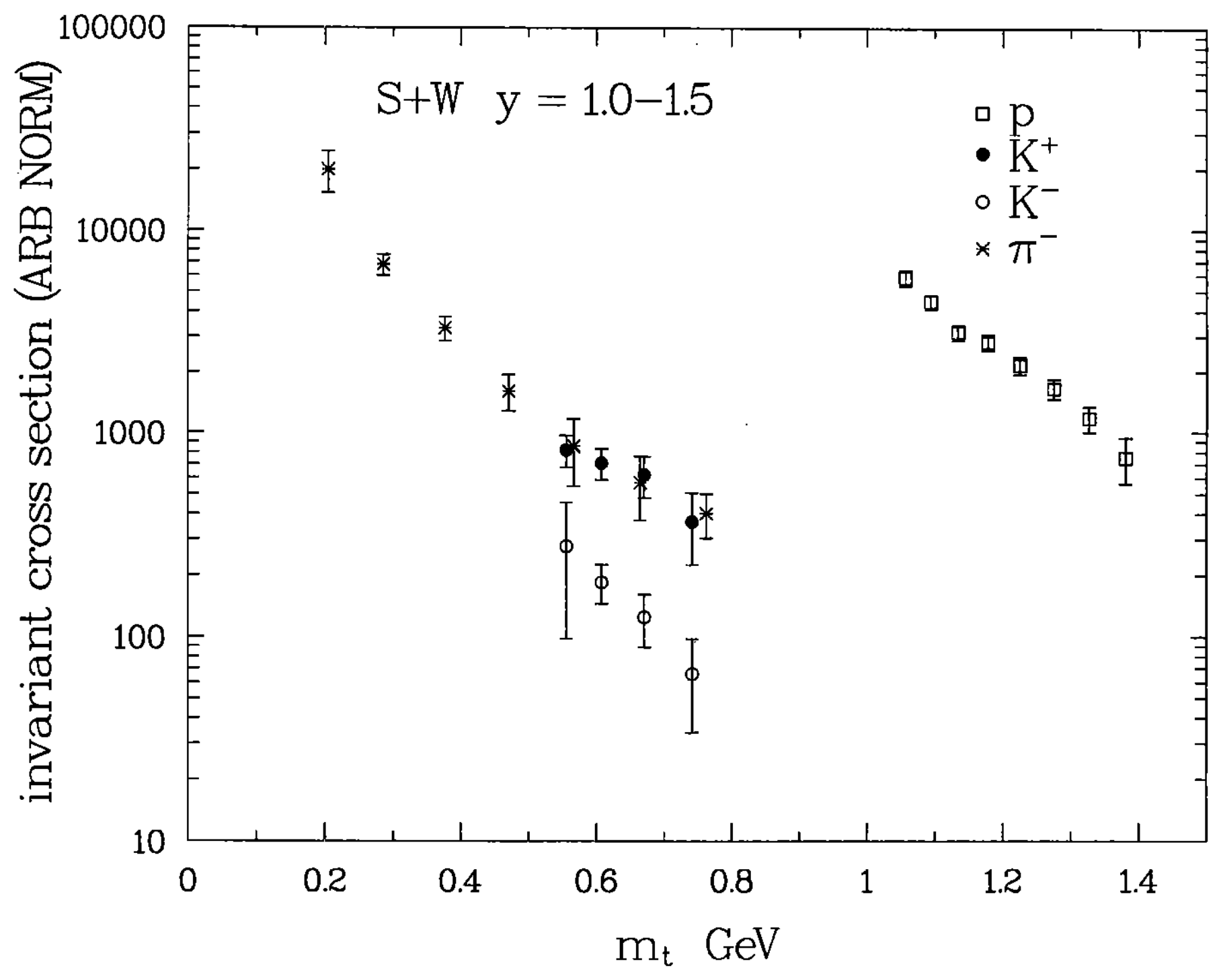

Fig. 3 